\title{
Involvement of Infralimbic Prefrontal Cortex but not Lateral Habenula in Dopamine Attenuation After Chronic Mild Stress
}

\author{
Jared L Moreines*, ${ }^{*, 2,3}$, Zoe L Owrutsky' and Anthony A Grace ${ }^{1,3}$ \\ 'Departments of Neuroscience, Psychology, and Psychiatry, Center for Neuroscience, University of Pittsburgh, Pittsburgh, PA, USA; ${ }^{2}$ Medical \\ Scientist Training Program, University of Pittsburgh School of Medicine, Pittsburgh, PA, USA; ${ }^{3}$ Center for the Neural Basis of Cognition, Carnegie \\ Mellon University, Pittsburgh, PA, USA
}

\begin{abstract}
Emerging evidence supports a role for dopamine in major depressive disorder (MDD). We recently reported fewer spontaneously active ventral tegmental area (VTA) dopamine neurons (ie, reduced dopamine neuron population activity) in the chronic mild stress (CMS) rodent model of MDD. In this study, we examined the role of two brain regions that have been implicated in MDD in humans, the infralimbic prefrontal cortex (ILPFC) — that is, rodent homolog of Brodmann area 25 (BA25), and the lateral habenula (LHb) in the CMSinduced attenuation of dopamine neuron activity. The impact of activating the ILPFC or LHb was evaluated using single-unit extracellular recordings of identified VTA dopamine neurons. The involvement of each region in dopamine neuron attenuation following 5-7 weeks of CMS was then evaluated by selective inactivation. Activation of either ILPFC or LHb in normal rats potently suppressed dopamine neuron population activity, but in unique patterns. ILPFC activation selectively inhibited dopamine neurons in medial VTA, which were most impacted by CMS. Conversely, LHb activation selectively inhibited dopamine neurons in lateral VTA, which were unaffected by CMS. Moreover, only ILPFC inactivation restored dopamine neuron population activity to normal levels following CMS; LHb inactivation had no restorative effect. These data suggest that, in the CMS model of MDD, the ILPFC is the primary driver of diminished dopamine neuron responses. These findings support a neural substrate for ILPFC/BA25 linking affective and motivational circuitry dysfunction in MDD. Neuropsychopharmacology (2017) 42, 904-913; doi:I0.1038/npp.2016.249; published online 7 December 2016
\end{abstract}

\section{INTRODUCTION}

Depression has been classically associated with dysfunction within the serotonin system, based primarily on the mode of action of antidepressant drugs (Carlsson, 1976). However, acutely increasing serotonin levels fail to alleviate symptoms, and antidepressant drugs require 8-12 weeks of treatment to be efficacious (Ressler and Nemeroff, 2000). In contrast, dopamine system dysfunction contributes to two key characteristics of depression: anhedonia (Treadway and Zald, 2011) and amotivation (Salamone and Correa, 2012). As a consequence, the dopamine system has garnered increasing attention for its role in depression (Nestler and Carlezon, 2006).

Dopamine neurons can fire in two distinct patterns, a spontaneous irregular tonic pattern and a more selectively recruited phasic burst pattern, the latter proposed to be the salient output of the system signaling behaviorally relevant stimuli (Grace and Bunney, 1984a, b). Because dopamine neurons must be spontaneously active to burst fire, the amount of baseline tonic activity represents the gain, or level

* Correspondence: IL Moreines, Department of Neuroscience, Center for Neuroscience, University of Pittsburgh, A210 Langley Hall, Pittsburgh, PA 15260, USA, Tel: 412624 7332, Fax: 412624 9198, E-mail: moreines.jared@medstudent.pitt.edu

Received 3 August 2016; revised 25 October 2016; accepted 27 October 20 16; accepted article preview online 4 November 2016 of amplification, of the phasic response (Lodge and Grace, 2006), with tonic firing regulated by a polysynaptic circuit leading to the ventral pallidum (Grace et al, 2007). In psychiatric disorders, aberrant functioning of this afferent control system is the more common source of dopamine system dysfunction, rather than a primary pathology of the dopamine neurons per se (Grace, 2016).

Using two distinct animal models of depression, we have recently demonstrated deficits in tonic dopamine neuron activity, expressed as reduced population activity of ventral tegmental area (VTA) dopamine neurons (Belujon and Grace, 2014; Chang and Grace, 2014). Moreover, we identified critical involvement of the ventral pallidum and basolateral amygdala in driving the reduced dopamine neuron population activity observed in rats exposed to the chronic mild stress (CMS) rodent depression model (Chang and Grace, 2014).

A recent surge of clinical and translational research has identified multiple additional circuit nodes potentially involved in depression-related dopamine system dysregulation. Brodmann area 25 (BA25) is homologous with the rodent infralimbic prefrontal cortex (ILPFC) (Heilbronner et al, 2016) and is widely considered to be a critical hub in depression neurocircuitry (Drevets, 1999; Mayberg, 2003). Nearly any form of therapeutically effective treatment for depression is associated with reversal of hypermetabolism in BA25 (Mayberg, 2009). In recent studies of normal rodents, 
activating ILFPC reduced dopamine neuron activity (Patton et al, 2013) as well as downstream network effects throughout the striatum that correlated with diminished reward-seeking behavior (Ferenczi et al, 2016). However, these experiments did not use depression animal models.

The lateral habenula ( $\mathrm{LHb}$ ) has also garnered attention for a possible role in depression (Hikosaka, 2010). Clinical data on the LHb are limited owing to its difficulty to image (Lawson et al, 2013); however, it has shown promise as a target for deep brain stimulation for intractable depression (Sartorius et al, 2010). Interest in the region emerged primarily from its role in the learned helplessness model of depression (Henn and Vollmayr, 2005; Li et al, 2011). When activated acutely, the $\mathrm{LHb}$ potently inhibits dopamine neuron firing (Ji and Shepard, 2007) and induces a transient aversive state (Stamatakis and Stuber, 2012) because of reduced synaptic dopamine levels (Hikosaka et al, 2008). However, the impact of persistent $\mathrm{LHb}$ activation on the population activity of dopamine neurons has not been assessed in normal rats or in depression animal models.

In the present study, we characterized the impact of ILPFC and $\mathrm{LHb}$ activation on dopamine neuron firing properties in normal rodents, and each region's involvement in the dopamine neuron inhibition induced by CMS. We found that ILPFC and LHb activation have divergent effects on dopamine neuron firing properties. Furthermore, only ILPFC inactivation following CMS was capable of restoring normal dopamine system activity levels.

\section{MATERIALS AND METHODS}

\section{Subjects}

All experiments were performed using adult male SpragueDawley rats (Envigo, Indianapolis, IN) weighing $\geq 300 \mathrm{~g}$, housed in pairs in a temperature- $\left(22^{\circ} \mathrm{C}\right)$ and humidity(47\%) controlled colony room (lights ON 0700-1900 hours) with food and water available ad libitum, and acclimated to the facilities for 7 days. All procedures were performed in accordance with the National Institutes of Health Guide for the Care and Use of Laboratory Animals and were approved by the Institutional Animal Care and Use Committee of the University of Pittsburgh.

\section{CMS Procedure}

Animals receiving CMS were single-housed in individual cages within a dedicated room. CMS was delivered as described previously (Chang and Grace, 2014). Stressorsdelivered in the home cage-included periodic restricted access to food and water, cage tilt, damp bedding, continuous overnight illumination, intermittent paired housing with an unfamiliar cage mate, white noise (80-90 db), stroboscopic lighting, and predator odor. Each week, 3-4 stressors were delivered in a novel combination.

\section{Behavioral Assays}

The full behavioral impact of our CMS procedure has been detailed previously (Chang and Grace, 2014). Thus, for the present study, only behaviors necessary to verify the CMS effect were assessed. Animals housed on a reverse light cycle were weighed weekly. At the conclusion of CMS, animals were tested for immobility on the forced swim test (FST). Owing to differences in sensitivity and behavioral profile of different rodent strains, we have found that the sucrose preference test is not a reliable indicator of dopamine system function specifically in Sprague-Dawley rats (Chang and Grace, 2014), unlike other strains such as Lister hooded, Long-Evans, or Wistar (Tye et al, 2013; Weiss, 1997; Willner et al, 1992, 1994).

FST procedure. The FST was performed following an established protocol (Slattery and Cryan, 2012) over two sequential days during the dark cycle, using plastic cylinder tubes (50 cm height; $20 \mathrm{~cm}$ diameter) filled water to a height of $30 \mathrm{~cm}\left(23-25^{\circ} \mathrm{C}\right)$. On day 1 , the rat was placed in the water for $15 \mathrm{~min}$ to eliminate nonspecific behaviors. On day $2(24 \mathrm{~h}$ later), the rat was placed in the water for $5 \mathrm{~min}$, and its behavior was captured by video for offline blinded scoring. The total time the rat spent immobile on day 2 was tabulated as an index of behavioral despair.

\section{Electrophysiological Recordings}

Surgery. Electrophysiological recordings were performed with light cycle counterbalanced across experimental groups. Rats were anesthetized with chloral hydrate $(400 \mathrm{mg} / \mathrm{kg}$; intraperitoneally), placed in a stereotaxic frame (David Kopf Instruments, Tujunga, CA), and maintained at $37^{\circ} \mathrm{C}$ using a thermocouple-controlled heating pad (Fine Science Tools, Foster City, CA). The skull was cleared of skin and fascia, and a partial craniectomy was performed to access the VTA and ILPFC or LHb using the following target coordinates referenced from bregma: VTA -5.3 to $5.7 \mathrm{~mm}$ posterior, +0.6 to $1.0 \mathrm{~mm}$ lateral, and -6.5 to $9.5 \mathrm{~mm}$ ventral (to brain surface); ILPFC $+3.2 \mathrm{~mm}$ anterior, $+0.5 \mathrm{~mm}$ lateral, and $-5.0 \mathrm{~mm}$ ventral; $\mathrm{LHb}-3.6 \mathrm{~mm}$ posterior, $+0.8 \mathrm{~mm}$ lateral, and v5.5 $\mathrm{mm}$ ventral.

Drug delivery. Microinfusions of drugs were delivered to the ILPFC or LHb using a 28-G stainless-steel cannula (Plastics One, Roanoake, VA) as described previously (Patton et al, 2013). All drugs were dissolved in Dulbecco's phosphate-buffered saline (dPBS; Sigma Aldrich, St Louis, $\mathrm{MO})$ as vehicle (VEH). NMDA ( $N$-methyl-D-aspartate (Sigma Aldrich), $0.75 \mu \mathrm{g}$ NMDA/0.5 $\mu \mathrm{l} \mathrm{dPBS}$ ) was used for activation, and tetrodotoxin (TTX (Sigma Aldrich), $1 \mu \mathrm{mol} /$ L) was used for inactivation, based on previous data establishing the neural effects of these dosages (Floresco et al, 2001; Patton et al, 2013). Infusion concentration was consistent for all regions, whereas volume was adjusted for brain region size $(0.5 \mu \mathrm{l}$ for ILPFC; $0.25 \mu \mathrm{l}$ for $\mathrm{LHb})$ and infused at a rate of $0.25 \mu \mathrm{l} / \mathrm{min}$ (total infusion time $2 \mathrm{~min}$ for ILPFC, $1 \mathrm{~min}$ for LHb). The cannula was left in place $>5$ min following infusion to allow for adequate diffusion.

VTA single unit recordings. Twenty minutes following drug microinfusion into LHb or ILPFC, single unit electrophysiological recordings of identified VTA dopamine neurons were performed. Single barrel electrodes were constructed from $2 \mathrm{~mm}$ diameter borosilicate capillary tubes (World Precision Instruments, Sarasota, FL) using a vertical 
a

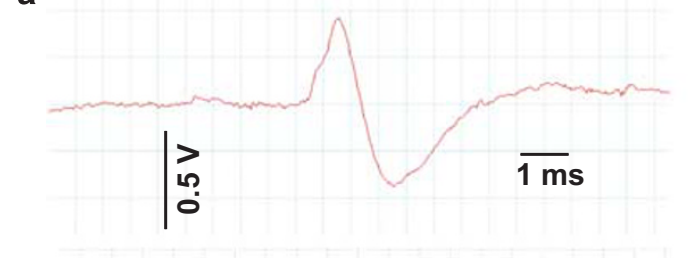

b

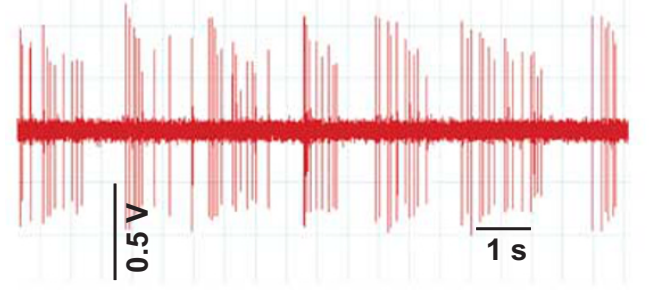

C

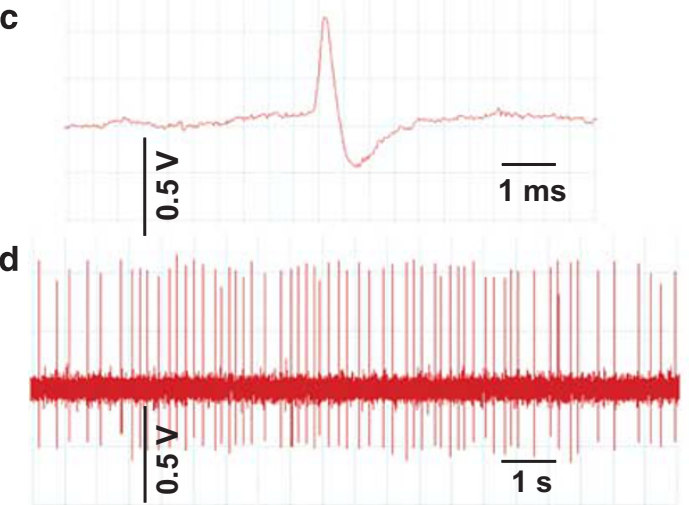

e

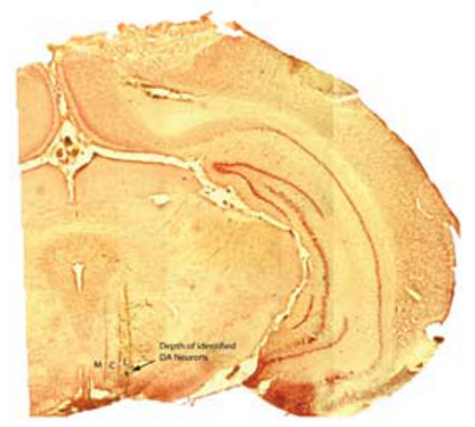

$\mathbf{f}$

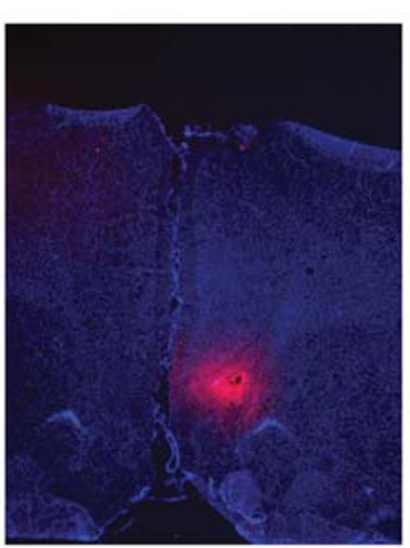

Figure I Dopamine recording procedure and microinfusion diffusion estimate. (a) Sample trace of dopamine neuron recording demonstrating key action potential features including long duration and biphasic ascending phase. (b) Sample recording of ventral tegmental area (VTA) dopamine neuron showing characteristic irregular, slow firing rate, mixed with sporadic periods of burst firing. (c) Sample trace of a neuron identified as non-dopaminergic. Note the short duration action potential. (d) Sample recording of a VTA neuron identified as non-dopaminergic. Note the comparatively more regular firing pattern. (e) Locations of medial (M), central (C), and lateral $(L)$ subregions of VTA. Blue dot in lateral track indicates the depth at which dopamine neurons were found in this animal. (f) Fluorescent microscope image from infusion of fluorescently tagged marker into infralimbic prefrontal cortex (ILPFC) used to estimate diffusion of subsequent drug microinfusions. LHb, lateral habenula.

electrode puller (Narishige, Tokyo, Japan), broken to a target of 6-10 M $\Omega$ under microscopic control, and filled with $2 \%$ Chicago Sky Blue (Sigma-Aldrich) dissolved in $2 \mathrm{M}$ saline. Signal was acquired using an amplifier (Fintronics, Orange, CT) with open filter settings ( $50 \mathrm{~Hz}$ low cutoff, $16 \mathrm{kHz}$ high cutoff, $1000 \times$ gain) and displayed on an oscilloscope (B\&K Precision, Yorba Linda, CA) with a signal fed to a computer running Lab Chart 7 (AD Instruments, Sydney, NSW, Australia). Units were recorded when signal-to-noise ratio exceeded $3: 1$.

Dopamine neuron identification. Electrodes were lowered into the VTA using a manual hydraulic microdrive (Kopf Instruments) and dopamine neurons were identified using well-established criteria including location, slow, irregular firing pattern, and long duration, variable shape biphasic action potential waveform $(>2.2 \mathrm{~ms})$, and half-width ( $>1.1 \mathrm{~ms}$ ) (Grace and Bunney, 1983; Ungless and Grace, 2012) (Figures 1a-d). Each dopamine neuron identified was recorded for $3 \mathrm{~min}$ ( $1 \mathrm{~min}$ minimum). Three parameters of dopamine neuron firing were calculated: (1) the number of dopamine neurons identified in each track (ie, population activity averaged over nine tracks), (2) firing rate, and (3) proportion of spikes occurring as burst firing (\%SIB) in which the burst onset was defined as two spikes with $\leq 80 \mathrm{~ms}$ interspike interval and termination by $>160 \mathrm{~ms}$ interspike interval (Grace and Bunney, 1984a).

VTA sampling. The VTA was sampled in nine sequential electrode tracks separated by $0.2 \mathrm{~mm}$ and arranged in a predetermined grid pattern to assess the distribution of dopamine neuron activity across the medial-lateral extent of the A10 region (Figure 1e). The 'Medial' dopamine neurons targeted in this paper include those located in the lateral-most aspects of the interfascicular and central linear nuclei and medial aspect of the paranigral $(\mathrm{PN})$ nucleus. 'Central' VTA sampled mostly from the PN nucleus and the medial aspect of the parabrachial pigmented (PBP) area. 'Lateral' VTA centered on the lateral-most aspects of the PBP area. This procedure was developed to sample dopamine neurons with a variety of different projection targets (Ikemoto, 2007), and has been published by us in multiple prior studies (Valenti et al, 2011, 2012). In order for a rat to be included in the final sample, data must have been available from all three VTA locations (ie, medial, central, and lateral). 


\section{Histology}

At the conclusion of recordings, final electrode placement was marked by electrophoretic ejection of dye. Rats were then overdosed with additional chloral hydrate, decapitated, and brains removed. Brains were fixed in $8 \%$ paraformaldehyde, followed by $25 \%$ sucrose for cryoprotection, sectioned using a cryostat (Leica, Buffalo Grove, IL) into $60 \mu \mathrm{m}$ coronal slices, mounted onto gelatin-chromalum-coated glass slides, and stained with cresyl violet and neutral red to check placements of recording electrodes and infusion cannulae tips. Extent of drug diffusion from ILPFC and LHb microinfusions was estimated from pilot infusions of an identical volume of fluorescent muscimol (TMR-X BODIPY, Fisher, Waltham, MA; Figure 1f). To ensure adequate and repeatable VTA sampling across animals, rats were excluded if VTA tracks were: (1) not observed laterally beyond the interfascicular and central linear nuclei, (2) located lateral to the PBP (ie, encroaching on the substantia nigra), or (3) only visible anterior to the $\mathrm{PN}$ or posterior to the PBP nucleus.

\section{Data Analysis}

Individual neuron data was analyzed by Lab Chart to identify spike time courses and exported to Neuroexpolorer (Nex Technologies, Madison, AL) to calculate firing rate and burst firing. Population activity (ie, cells per track; CPT) was averaged within each animal followed by across animals in each group, whereas neuron firing rate and burst activity were averaged across animals in a group, considering each cell as an independent replicate. Data were analyzed using SPSS 23 (IBM, Armonk, NY). All data sets were compared with the normal Gaussian distribution using the Kolmogorov-Smirnov and Shapiro-Wilk tests. Data sets that deviated from the normal distribution were analyzed with MannWhitney $U$-test or Kruskal-Wallis $H$-test, followed by Dunn's post hoc tests as appropriate. Normally distributed data were analyzed using independent sample two-tailed $t$ tests or ANOVA followed by Tukey's post hoc tests as appropriate.

\section{RESULTS}

\section{ILPFC Activation Attenuates Medial VTA Dopamine Neuron Activity}

ILPFC activation by local infusion of NMDA markedly reduced the number of spontaneously active VTA dopamine neurons recorded per electrode track $(\mathrm{VEH} 1.1 \pm 0.12 \mathrm{CPT}$, $n=8$; NMDA $0.76 \pm 0.038 \mathrm{CPT}, n=11 ; t_{8.4}=2.4, p=0.040$; Figure 2a), consistent with prior studies (Patton et al, 2013). ILPFC activation also induced a small but significant increase in average burst firing (VEH $21.6 \pm 3.2 \% \mathrm{SIB}$, $n=68 ;$ NMDA $27.7 \pm 3.2 \% \mathrm{SIB}, n=61 ; U=2.12, p=0.034$; Figure 2f). No change in average firing rate was observed (VEH $3.4 \pm 0.26 \mathrm{~Hz}, n=68$; NMDA $3.3 \pm 0.23 \mathrm{~Hz}, n=61$; $U=0.307, p=0.76$; Figure 2c).

Given evidence that the VTA is functionally segregated (Ikemoto, 2007), the data were analyzed according to location in medial, central, or lateral VTA. Population activity was profoundly reduced in medial VTA (VEH $1.3 \pm 0.12 \mathrm{CPT}, n=8 ; \mathrm{NMDA} 0.65 \pm 0.15 \mathrm{CPT}, n=11$; $t_{17}=3.4, p=0.0037$; Figure $2 \mathrm{~b}$ ), but not affected significantly in central and lateral portions (two-way ANOVA location $x$ drug interaction, $\left.\mathrm{F}_{(2,34)}=3.35, p=0.047\right)$. In contrast, elevated bursting was found to be isolated to neurons in lateral VTA (VEH $20.5 \pm 6.7 \% \mathrm{SIB}, \quad n=18 ; \quad \mathrm{NMDA}$ $39.0 \pm 5.4 \% \mathrm{SIB}, n=16 ; \quad U=2.48, p=0.012$; Figure $2 \mathrm{~g}$ and Supplementary Table 1). The frequency distribution of bursting for these neurons suggested that ILPFC activation drove a previously non-bursting population (ie, $0 \% \mathrm{SIB}$ ) into a higher bursting state (Figure $2 \mathrm{~h}$ ). ILPFC activation did not alter average dopamine neuron firing rate, either overall or in any VTA subregion (Figure $2 \mathrm{~d}$ and Supplementary Table 1).

\section{LHb Activation Induces Unique Subregion-Specific Effects on VTA Activity}

Local infusion of NMDA into the $\mathrm{LHb}$ caused a marked reduction in dopamine neuron population activity (VEH $1.0 \pm 0.087 \mathrm{CPT}, n=10 ; \mathrm{NMDA} 0.68 \pm 0.070 \mathrm{CPT}, n=12$; $t_{20}=3.1, p=0.0053$; Figure 3a) along with a modest increase in average VTA dopamine neuron firing rate $(\mathrm{VEH}$ $3.3 \pm 0.24 \mathrm{~Hz}, n=73$; NMDA $4.1 \pm 0.25 \mathrm{~Hz}, n=69 ; U=2.6$, $p=0.008$; Figure $3 c$ ). There was no change in average amount of burst activity (VEH 26.2 $\pm 3.0 \% \mathrm{SIB}, n=73$; NMDA $26.3 \pm 2.9 \%$ SIB, $n=69 ; U=0.58, p=0.56$; Figure $3 f$ ).

In marked contrast to the ILPFC, LHb activation potently and selectively decreased population activity of lateral VTA dopamine neurons, inducing a 70\% decrease (VEH $0.88 \pm 0.12 \mathrm{CPT}, n=10$, NMDA $0.26 \pm 0.063 \mathrm{CPT}, n=12$; $t_{20}=4.9, p=0.000092$; Figure $3 \mathrm{~b}$ ). Moreover, LHb activation induced a $39 \%$ increase in firing rate of medially located VTA dopamine neurons (VEH $3.3 \pm 0.24 \mathrm{~Hz}, n=29$; NMDA $4.7 \pm 0.36 \mathrm{~Hz}, n=32 ; U=2.8, p=0.005$; Figure $3 \mathrm{~d})$. Bursting was unaffected in all VTA subregions (Figure $3 g$ and Supplementary Table 2).

\section{CMS Impacts Primarily Medial and Central VTA}

To determine the relevance of ILPFC and LHb activity to dopamine system hypofunction in an animal model of depression, the regionally specific effects of CMS on VTA dopamine neuron firing, and how this compares with the impact of ILPFC and LHb activation, were assessed.

Following CMS, animals showed increased FST immobility (CON $173.4 \pm 23.38 \mathrm{~s}, n=8$, CMS $233.3 \pm 9.91 \mathrm{~s}, n=8$, $t_{14}=2.36, p=0.037$; Supplementary figure S1a) and reduced body weight gain (two-way ANOVA group $\times$ time Interaction $\mathrm{F}_{(5,36)}=3.7, p=0.0078$; Supplementary figure $\mathrm{S} 1 \mathrm{~b}$ ), consistent with prior studies (Chang and Grace, 2014). CMSexposed rats exhibited $>50 \%$ fewer spontaneously active VTA dopamine neurons (CON-VEH $1.3 \pm 0.091$ CPT, $n=12$, CMS-VEH $0.62 \pm 0.049$ CPT, $n=14 ; \quad H=22.4$, $p<0.0001$; Figure $4 \mathrm{a}$ ), with no change in dopamine neuron firing rate (CON-VEH $3.8 \pm 0.18 \mathrm{~Hz}, n=125$, CMS-VEH $3.7 \pm 0.24 \mathrm{~Hz}, n=82 ; H=6.0, p=0.11$; Figure $4 \mathrm{c}$ ) or bursting (CON-VEH $31 \pm 2.4 \%$ SIB, CMS-VEH $28 \pm 2.9 \%$ SIB, $H=2.9$, $p=0.40$; Figure $4 \mathrm{f}$ ), also consistent with prior studies (Chang and Grace, 2014). When examined with regard to VTA subregions, CMS-exposed rats showed $67 \%$ reductions in dopamine neuron population activity in medial VTA (CON-VEH $1.5 \pm 0.13$ CPT, $n=12$, CMS-VEH $0.55 \pm 0.084$ CPT, $n=14 ; H=21.3, p<0.0001$; Figure $4 \mathrm{~b}$ ) and $50 \%$ in 

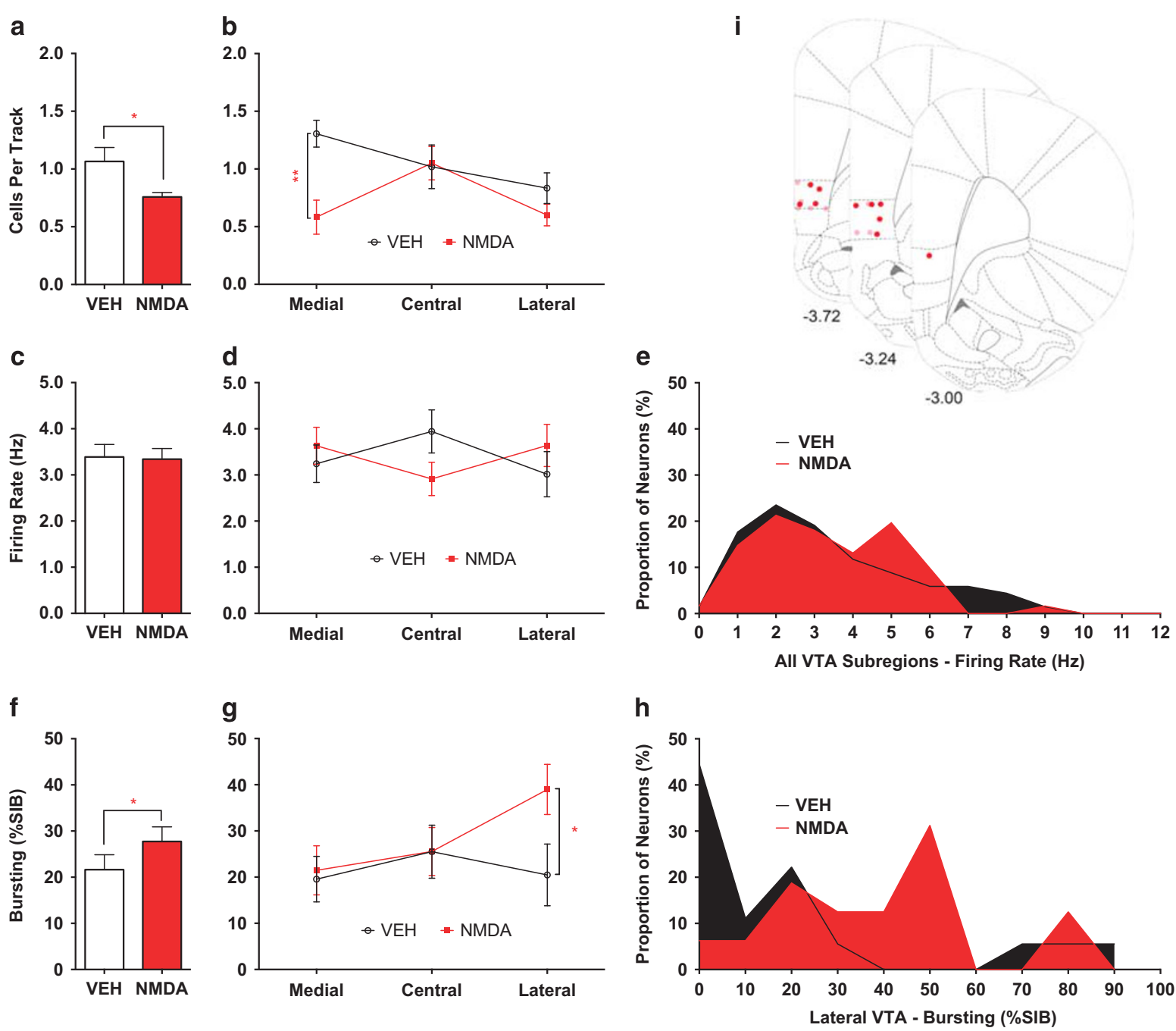

Figure 2 Activation of infralimbic prefrontal cortex (ILPFC) in normal rats. ILPFC activation selectively attenuated dopamine neuron population activity in the medial aspects of the ventral tegmental area (VTA). (a) Dopamine neuron population activity was reduced following microinfusion of N-methyl-Daspartate (NMDA) into the ILPFC. (b) This effect was significant only in medial VTA. (c-e) Average firing rate of identified dopamine neurons was not different between animals that received vehicle (VEH) infusions vs NMDA. (f) Average amount of bursting activity in identified dopamine neurons was increased in animals that received NMDA vs VEH. (g) This occurred via a select increase in bursting activity in dopamine neurons located in lateral VTA. (h) Examination of the histograms of lateral VTA dopamine neuron bursting levels for each infusion group suggested that NMDA drove lateral VTA dopamine neurons from a typically non-bursting state (ie, $0 \% \mathrm{SIB}$ ) into one in which more neurons showed moderate levels of bursting. (i) Location of NMDA (dark red circles) and VEH (light red circles) infusions into ILPFC. $* p<0.05$ and $* * p<0.01$; \%SIB, percent spikes in burst.

central VTA (CON-VEH $1.4 \pm 0.17 \mathrm{CPT}, n=12$, CMS-VEH $0.77 \pm 0.10 \mathrm{CPT}, n=14 ; H=14.5, p=0.012$; Figure $4 \mathrm{~b}$ ), whereas lateral VTA was not significantly impacted (CON-VEH $0.92 \pm 0.16$ CPT, $n=12$, CMS-VEH $0.53 \pm 0.10$ $\mathrm{CPT}, n=14 ; H=10.2, p=0.12$; Figure $4 \mathrm{~b})$. No changes were observed following CMS in average dopamine neuron firing rate or bursting activity in any VTA subregion (Figures $4 \mathrm{c}-\mathrm{h}$ and Supplementary Table 3).

\section{ILPFC but not LHb Inactivation Restores Dopamine Activity in CMS-Exposed Rats}

The involvement of the ILPFC and LHb in CMS-induced attenuation of VTA dopamine neuron activity was assessed by inactivating each region with TTX in CMS-exposed rats (Figure 4). ILPFC inactivation was found to restore dopamine neuron population activity to a level comparable to that of normal rats (CON-VEH $1.3 \pm 0.091 \mathrm{CPT}, n=12$, CMS-ILPFC-TTX $1.1 \pm 0.078$ CPT, $n=10 ; H=3.9, p>0.99$; Figure 4a). In contrast, LHb inactivation did not affect the reduced VTA dopamine neuron population activity (CON-VEH $1.3 \pm 0.091 \quad$ CPT, $n=12$, CMS-LHb-TTX $0.57 \pm 0.057 \mathrm{CPT}, n=8 ; H=31.3, p<0.0001$; Figure $4 \mathrm{a}$ ).

TTX infusion into the ILPFC of CMS-exposed rats also normalized dopamine neuron population activity in medial (CON-VEH $1.5 \pm 0.13$ CPT, $n=12$, CMS-ILPFC-TTX $1.1 \pm 0.14 \mathrm{CPT}, \quad n=10 ; \quad H=6.6, p>0.99)$ and central (CON-VEH $1.4 \pm 0.17$ CPT, $n=12$, CMS-ILPFC-TTX $1.1 \pm 0.16 \mathrm{CPT}, n=10 ; H=6.2, p=0.77)$ VTA to levels comparable to control animals (Figure $4 \mathrm{~b}$ ). A concurrent small reduction in average firing rate of medial dopamine neurons was observed (CON-VEH $3.8 \pm 0.27 \mathrm{~Hz}, n=50$, CMS-ILPFC-TTX $2.6 \pm 0.27 \mathrm{~Hz}, n=28 ; H=20.8, p=0.023$ ), possibly because of the emergence of a new population of 


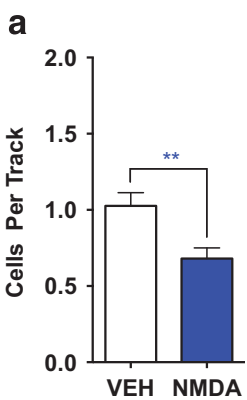

\section{b}
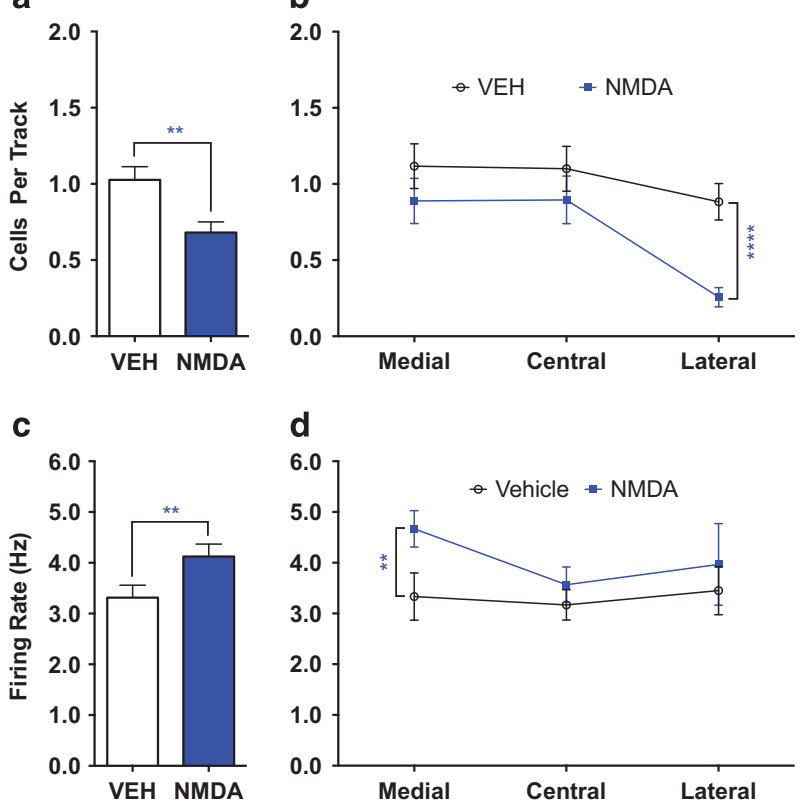

\section{d}

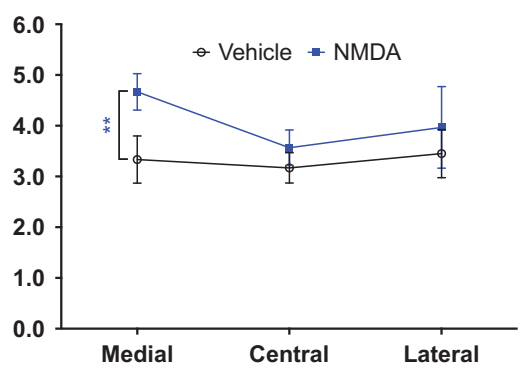

f

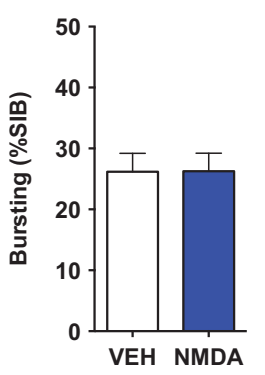

i
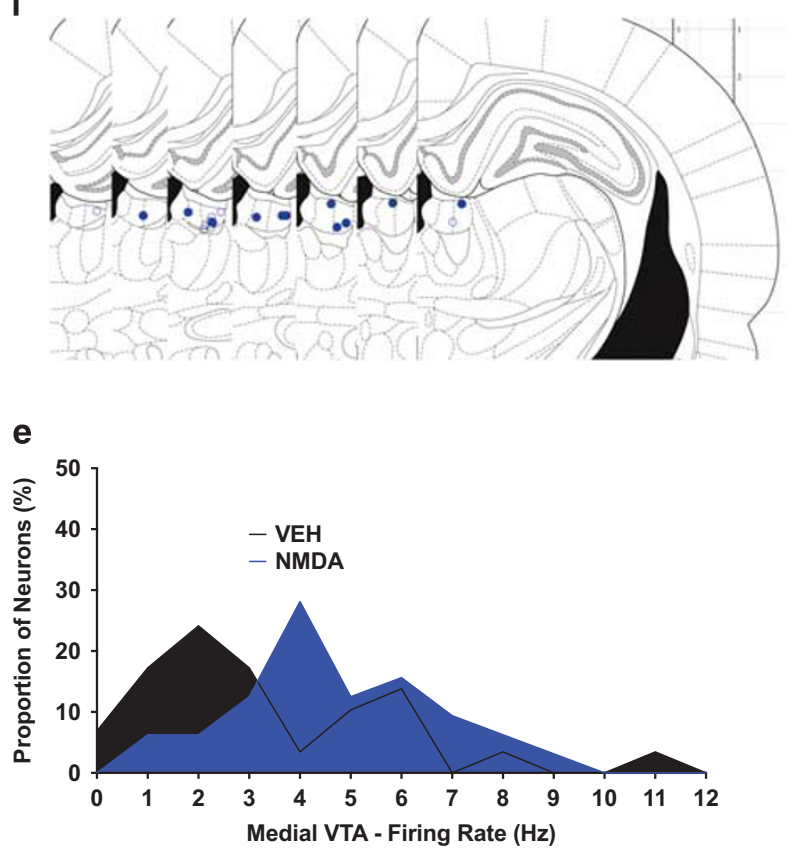

h

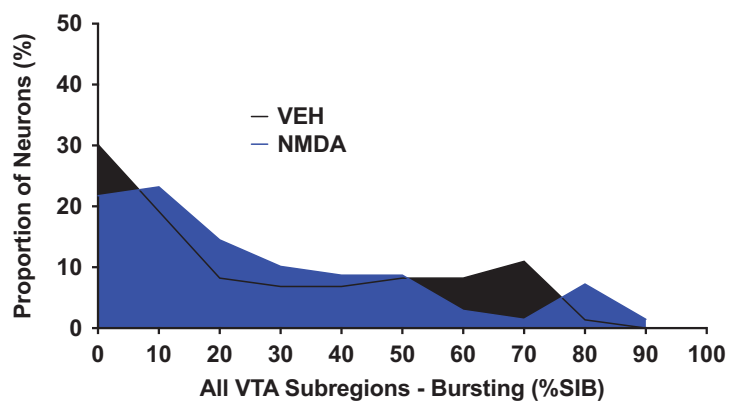

Figure 3 Activation of lateral habenula $(\mathrm{LHb})$ in normal rats. $\mathrm{LHb}$ activation selectively attenuated dopamine neuron population activity in the lateral aspects of the ventral tegmental area (VTA). (a) Dopamine neuron population activity was reduced following infusion of N-methyl-D-aspartate (NMDA) into the LHb. (b) This occurred via selective reduction in lateral VTA dopamine neuron population activity. (c) Average firing rate of identified dopamine neurons was higher in NMDA-infused rats vs vehicle $(V E H)$. ( $d$ and e) This effect was driven by a selective increase in the average firing rate of dopamine neurons located in medial VTA following NMDA infusion in LHb. (f-h) No changes occurred in average amount of dopamine neuron bursting activity following infusion of NMDA. (i) Location of NMDA (closed blue circles) and VEH (open blue circles) infusions into LHb. $* * p<0.0$ I and ***** $p<0.000 \mathrm{I}$. \%SIB, percent spikes in burst.

slow-firing dopamine neurons in medial VTA following TTX infusion into the ILPFC (Figures 4c-e). In contrast, TTX infusion into the LHb exerted no effect on VTA dopamine neuron population activity or firing parameters in CMSexposed rats in any subregion of the VTA (Figure 4 and Supplementary Table 3). These data demonstrate a critical role for ILPFC in CMS-induced reductions in dopamine neuron population activity.

\section{DISCUSSION}

In this study, activation of either the ILPFC or the LHb in normal animals reduced the population activity of VTA dopamine neurons (ie, the number of dopamine neurons spontaneously active). Whereas ILPFC activation exerted a greater inhibitory effect on medial VTA dopamine neurons, the LHb selectively inhibited lateral VTA dopamine neurons (Figure 5). In rats exposed to CMS, the reduction in dopamine neuron population activity was preferential to medial and central VTA. ILPFC inactivation restored dopamine neuron population activity to a normal level, whereas LHb inactivation did not affect this parameter.

Following some of the manipulations, in addition to changes in population activity, there were also small but significant changes in firing rate and bursting. We have reported previously that DA neurons must be spontaneously active to respond to behaviorally relevant, phasic inputs (Floresco et al, 2003; Lodge and Grace, 2006). Therefore, a change in population activity better reflects the responsivity of the system, and is altered in several disease states (Grace, 2016). ILPFC activation in normal rats reduced dopamine population activity medially but increased the tendency of lateral neurons to burst fire. Because the majority of dopamine neurons in medial VTA project to accumbens shell, reducing the active number would be expected to reduce markedly the response of ventromedial accumbensprojecting DA neurons to reward-related stimuli (Grace, 2016) as only spontaneously active dopamine neurons are 

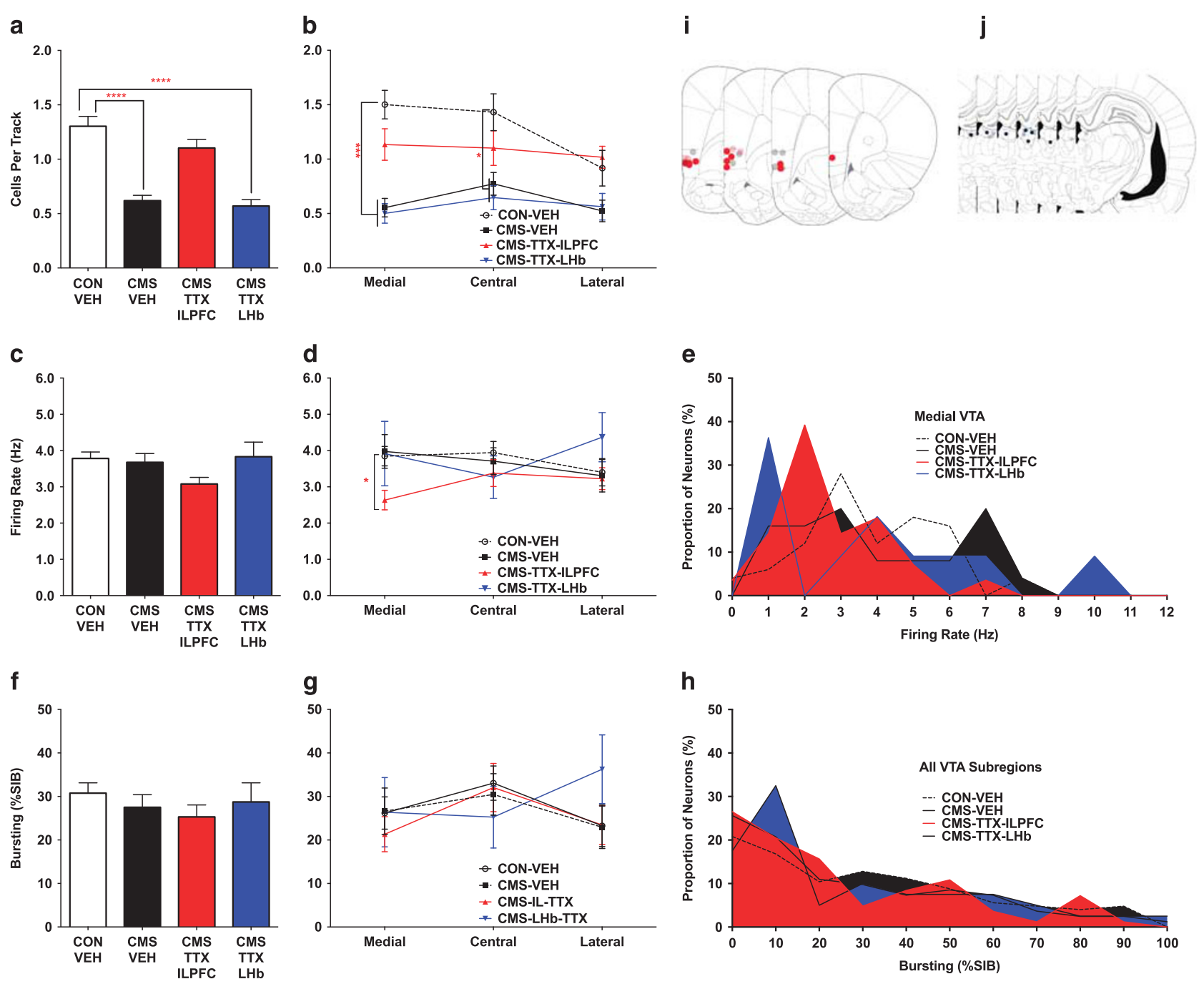

Figure 4 Inactivation of infralimbic prefrontal cortex (ILPFC) or lateral habenula (LHb) following chronic mild stress. Only ILPFC inactivation restored ventral tegmental area (VTA) dopamine neuron activity in chronic mild stress (CMS)-exposed rats. (a) Rats that underwent CMS showed lower VTA dopamine neuron population activity compared with rats housed in control (CON) conditions. Inactivation of ILPFC using microinfusion of tetrodotoxin (TTX) restored normal dopamine neuron population activity, whereas inactivation of LHb using microinfusion of TTX had no impact on VTA dopamine neuron population activity. (b) The reduction in VTA dopamine neuron population activity following CMS was greatest in medial and central VTA subregions, whereas no significant change in dopamine population activity was observed in lateral VTA. Inactivation of the ILPFC using TTX resulted in normalization of dopamine neuron population activity in medial and central VTA subregions. (c and d) While VTA-wide average dopamine neuron firing rates did not differ between groups, a select reduction in the average firing rate of dopamine neurons located in medial VTA was observed in CMS-TTX-ILPFC rats compared with CON. (e) The histogram of medial VTA dopamine neuron firing rates suggests an increase in the number of dopamine neurons firing at a slower rate following TTX microinfusion into the ILPFC in CMS-exposed rats. ( $f-h)$ No changes in the amount of bursting activity in identified dopamine neurons occurred in any group or VTA subregion. ( $i$ and j) Location of microinfusions for CON-vehicle (CON-VEH) (gray circles), CMS-VEH (light red and blue circles), CMS-TTX-ILPFC (dark red circles), and CMS-TTX-LHb (dark blue circles). $* p<0.05$, **** $p<0.00$ I, and ***** $p<0.000$ I. \%SIB, percent spikes in burst.

capable of responding to signals with burst firing. This may underlie the reduced motivation for reward in depressed individuals, as Ferenczi et al (2016) showed that activation of the ILPFC-induced depression-related behavior in normal rats that was associated with functional connectivity changes between the ILPFC and ventral striatum. In contrast, the increased tendency to burst fire was observed in lateral VTA dopamine neurons, which project more strongly to associative striatum, although the number of spontaneously active neurons did not change. Although speculative, this tonic change in bursting could reflect changes in plasticity with respect to the salience of environmental stimuli, contributing to the well-documented affective bias and hypersensitivity to negative valence stimuli and feedback in depression (Clark et al, 2009).

Following CMS, inactivation of the ILPFC restored the number of spontaneously active dopamine neurons to normal levels, while reducing the average firing rate of medial dopamine neurons, because of an increase in the number of slower firing dopamine neurons (Figure 4e). While changes in firing rate may engender a change in baseline DA levels in the accumbens, the impact on responsivity to stimuli would be comparatively minimal and likely to undergo homeostatic compensation.

Intriguingly, ILPFC inactivation in CMS-exposed rats restored normal dopamine population activity in both 

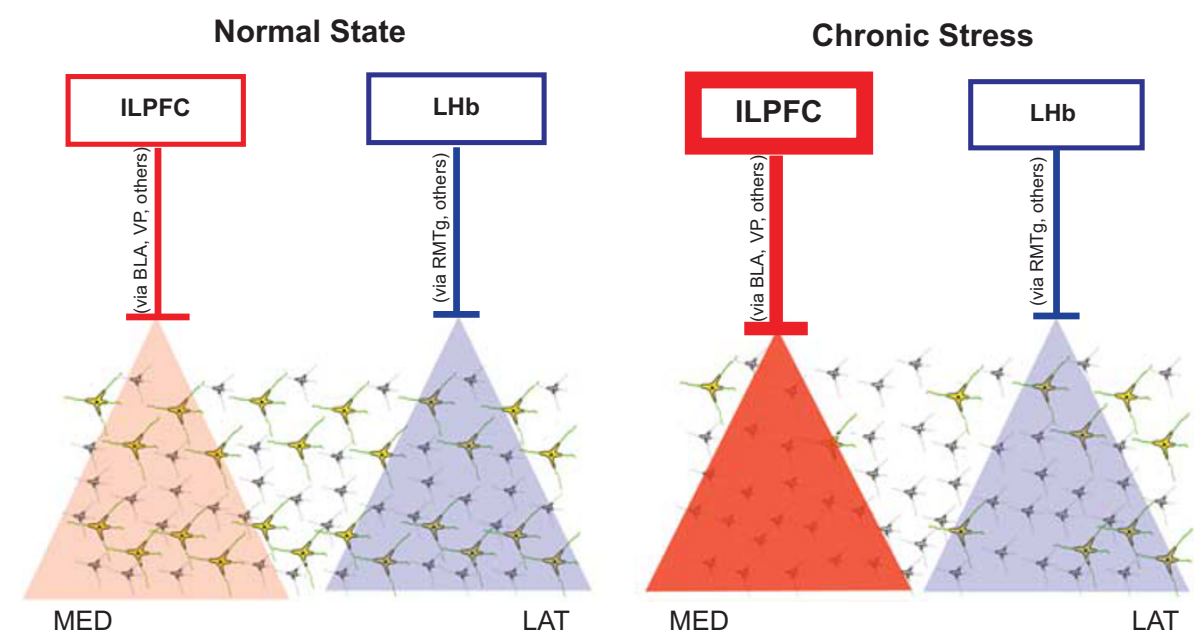

Figure 5 One potential circuit diagram proposed to account for infralimbic prefrontal cortex (ILPFC) and lateral habenula (LHb) regulation of dopamine neuron population activity in normal and stressed states. In the normal state, afferent regions are functioning at baseline levels, leading to baseline levels of ventral tegmental area (VTA) dopamine neuron population activity. When activated, the ILPFC preferentially inhibits dopamine neurons located in medial VTA, which tend to project more to the reward- and motivation-related areas of the nucleus accumbens, whereas the LHb preferentially inhibits dopamine neurons located in lateral VTA, which project more to cognitive/decision-related dorsomedial striatal regions (see Ikemoto, 2007, Figure I2 for a review of anatomy). Chronic mild stress (CMS) involves ILPFC-driven inhibition of dopamine neurons, as its inactivation restored normal levels of dopamine neuron population activity. BLA, basolateral amygdala; LAT, lateral VTA; MED, medial VTA; RMTg, rostromedial tegmentum; VP, ventral pallidum; VTA, ventral tegmental area.

medial and central VTA, while ILPFC activation in normal rats inhibited only medial VTA population activity. In normal rats, ILPFC inactivation increases dopamine neuron population activity through indirect activation of the ventral subiculum of the hippocampus via the nucleus reunions (Patton et al, 2013; Zimmerman and Grace, 2016). Thus, inhibition of ILPFC in the CMS-exposed rats in the present paper (or inhibition of BA25 in patients with depression such as via DBS) may engage distant downstream brain networks that together work to restore normal dopaminemediated hedonic functions via a mechanism that may differ from simple reversal of abnormal plasticity per se.

$\mathrm{LHb}$ activation in normal rats reduced dopamine population activity laterally but increased firing rate medially. Inhibition of lateral dopamine neurons has been shown to alter reward expectation and decision-making (Eshel et al, 2016), supporting a role for LHb-mediated inhibition of these neurons impacting cognitive tasks (Stopper and Floresco, 2014). Conversely, the small increase in medial dopamine neuron firing rate with LHb activation likely reflects a known, small, monosynaptic glutamatergic projection from the $\mathrm{LHb}$ to cortically projecting dopamine neurons in medial VTA (Lammel et al, 2012). Although this disynaptic projection was shown to be aversive functionally (assessed by conditioned place avoidance), increased activity of cortically projecting dopamine neurons has actually been shown to be protective against developing depression (Chaudhury et al, 2013). In CMS-exposed rats, LHb inactivation had no effect. Importantly, LHb activation has previously been shown to be aversive (Stamatakis and Stuber, 2012), but never to be prodepressive in normal animals (ie, without a genetic predisposition to helplessness). Moreover, recent neuroimaging data questions whether heightened LHb activity actually drives responses in depressed humans (Lawson et al, 2016).
These results add to the accumulating evidence supporting a major role of the dopamine system in stress and depression. Dopamine lesions, antagonists, or direct optogenetic attenuation of dopamine neurons reduces reward pursuit similar to that seen in depressed patients with anhedonia, whereas rats that have received CMS show a reversal of behavioral despair by optogenetic activation of VTA dopamine neurons (Tye et al, 2013). While this may at first appear inconsistent with studies using the chronic social defeat (CSD) model, in which dopamine system activation facilitates defeat-mediated depression (Chaudhury et al, 2013), this may have more to do with the relative timing of the interventions. Thus, in each of the studies with CMS or $\mathrm{LH}$, dopamine neuron activity was measured $>24 \mathrm{~h}$ after removal of the stressor. In contrast, activation of accumbalprojecting dopamine neurons with social defeat during the induction phase increased the susceptibility of mice to defeat induced depression, consistent with an established role for these neurons in acute stress (Valenti et al, 2011). These collective data are highly consistent with a model in which stress-induced dopamine neuron activation is followed by a much longer attenuation of dopamine neuron activity, as has been observed $24 \mathrm{~h}$ after dopamine system activation by restraint (Chang and Grace, 2013) or amphetamine (Belujon et al, 2016), and following chronic cold stress (Moore et al, 2001; Valenti et al, 2012). This is consistent with Koob's opponent process model, in which dopamine activation is followed by a long-duration compensatory attenuation of the dopamine system (Koob et al, 1989). Thus, we observed the decrease in dopamine neuron activity following withdrawal of learned helplessness or CMS (Belujon and Grace, 2014; Chang and Grace, 2014), and Tye et al (2013) found that VTA dopamine neuron activation reversed the impact of CMS following withdrawal of the stressor, which was later 
replicated in the CSD depression model as well (Friedman et al, 2014).

In summary, the current data highlight distinct roles for the ILPFC and LHb in regulating VTA dopamine neurons in normal and chronic stress-exposed states. If this translates to major depressive disorder in humans, this also would provide a target site for therapeutic intervention within the ILPFC/BA25 to normalize dopamine neuron drive.

\section{FUNDING AND DISCLOSURE}

This work was supported by NIH Grants MH105199 (to JLM), MH101180 (to AAG), NS007433, and GM008208, as well as pilot funding from Lundbeck. Dr Grace receives consulting fees from Abbott, Alkermes, Asubio, Autofony, Dainippon Sumitomo, GSK, Johnson \& Johnson, Lilly, Lundbeck, Merck, Otsuka, Pfizer, Roche, and Takeda. He receives research funding from Lundbeck and Janssen. Moreines and Owrutsky reported no biomedical financial interests or potential conflict of interest.

\section{ACKNOWLEDGMENTS}

We thank Kathryn Gill, Pauline Belujon, Millie RinconCortes, Felipe Gomes, and Tomek Banasikowski for helpful discussion and guidance, and Niki MacMurdo and Christy Smolak for histology technical assistance.

\section{REFERENCES}

Belujon P, Grace AA (2014). Restoring mood balance in depression: ketamine reverses deficit in dopamine-dependent synaptic plasticity. Biol Psychiatry 76: 927-936.

Belujon P, Jakobowski NL, Dollish HK, Grace AA (2016). Withdrawal from acute amphetamine induces an amygdala-driven attenuation of dopamine neuron activity: reversal by ketamine. Neuropsychopharmacology 41: 619-627.

Carlsson A (1976). The contribution of drug research to investigating the nature of endogenous depression. Pharmakopsychiatr Neuro-Psychopharmakol 9: 2-10.

Chang CH, Grace AA (2013). Amygdala beta-noradrenergic receptors modulate delayed downregulation of dopamine activity following restraint. J Neurosci 33: 1441-1450.

Chang CH, Grace AA (2014). Amygdala-ventral pallidum pathway decreases dopamine activity after chronic mild stress in rats. Biol Psychiatry 76: 223-230.

Chaudhury D, Walsh JJ, Friedman AK, Juarez B, Ku SM, Koo JW et al (2013). Rapid regulation of depression-related behaviours by control of midbrain dopamine neurons. Nature 493: 532-536.

Clark L, Chamberlain SR, Sahakian BJ (2009). Neurocognitive mechanisms in depression: implications for treatment. Annu Rev Neurosci 32: 57-74.

Drevets WC (1999). Prefrontal cortical-amygdalar metabolism in major depression. Ann N Y Acad Sci 877: 614-637.

Eshel N, Tian J, Bukwich M, Uchida N (2016). Dopamine neurons share common response function for reward prediction error. Nat Neurosci 19: 479-486.

Ferenczi EA, Zalocusky KA, Liston C, Grosenick L, Warden MR, Amatya D et al (2016). Prefrontal cortical regulation of brainwide circuit dynamics and reward-related behavior. Science (New York, NY) 351: aac9698.

Floresco SB, Todd CL, Grace AA (2001). Glutamatergic afferents from the hippocampus to the nucleus accumbens regulate activity of ventral tegmental area dopamine neurons. J Neurosci 21: 4915-4922.

Floresco SB, West AR, Ash B, Moore H, Grace AA (2003). Afferent modulation of dopamine neuron firing differentially regulates tonic and phasic dopamine transmission. Nat Neurosci 6: 968-973.

Friedman AK, Walsh JJ, Juarez B, Ku SM, Chaudhury D, Wang J et al (2014). Enhancing depression mechanisms in midbrain dopamine neurons achieves homeostatic resilience. Science (New York, NY) 344: 313-319.

Grace AA (2016). Dysregulation of the dopamine system in the pathophysiology of schizophrenia and depression. Nat Rev Neurosci 17: 524-532.

Grace AA, Bunney BS (1983). Intracellular and extracellular electrophysiology of nigral dopaminergic neurons-1. Identification and characterization. Neuroscience 10: 301-315.

Grace AA, Bunney BS (1984a). The control of firing pattern in nigral dopamine neurons: burst firing. J Neurosci 4: 2877-2890.

Grace AA, Bunney BS (1984b). The control of firing pattern in nigral dopamine neurons: single spike firing. J Neurosci 4: 2866-2876.

Grace AA, Floresco SB, Goto Y, Lodge DJ (2007). Regulation of firing of dopaminergic neurons and control of goal-directed behaviors. Trends Neurosci 30: 220-227.

Heilbronner SR, Rodriguez-Romaguera J, Quirk GJ, Groenewegen HJ, Haber SN (2016). Circuit-based corticostriatal homologies between rat and primate. Biol Psychiatry 80: 509-521.

Henn FA, Vollmayr B (2005). Stress models of depression: forming genetically vulnerable strains. Neurosci Biobehav Rev 29: 799-804.

Hikosaka O (2010). The habenula: from stress evasion to valuebased decision-making. Nat Rev Neurosci 11: 503-513.

Hikosaka O, Sesack SR, Lecourtier L, Shepard PD (2008). Habenula: crossroad between the basal ganglia and the limbic system. J Neurosci 28: 11825-11829.

Ikemoto S (2007). Dopamine reward circuitry: two projection systems from the ventral midbrain to the nucleus accumbensolfactory tubercle complex. Brain Res Rev 56: 27-78.

Ji H, Shepard PD (2007). Lateral habenula stimulation inhibits rat midbrain dopamine neurons through a GABA(A) receptormediated mechanism. J Neurosci 27: 6923-6930.

Koob GF, Stinus L, Le Moal M, Bloom FE (1989). Opponent process theory of motivation: neurobiological evidence from studies of opiate dependence. Neurosci Biobehav Rev 13: 135-140.

Lammel S, Lim BK, Ran C, Huang KW, Betley MJ, Tye KM et al (2012). Input-specific control of reward and aversion in the ventral tegmental area. Nature 491: 212-217.

Lawson RP, Drevets WC, Roiser JP (2013). Defining the habenula in human neuroimaging studies. NeuroImage 64: 722-727.

Lawson RP, Nord CL, Seymour B, Thomas DL, Dayan P, Pilling S et al (2016). Disrupted habenula function in major depression. Mol Psychiatry (e-pub ahead of print).

Li B, Piriz J, Mirrione M, Chung C, Proulx CD, Schulz D et al (2011). Synaptic potentiation onto habenula neurons in the learned helplessness model of depression. Nature 470: 535-539.

Lodge DJ, Grace AA (2006). The hippocampus modulates dopamine neuron responsivity by regulating the intensity of phasic neuron activation. Neuropsychopharmacology 31: 1356-1361.

Mayberg HS (2003). Modulating dysfunctional limbic-cortical circuits in depression: towards development of brain-based algorithms for diagnosis and optimised treatment. $\mathrm{Br}$ Med Bull 65: 193-207.

Mayberg HS (2009). Targeted electrode-based modulation of neural circuits for depression. J Clin Invest 119: 717-725.

Moore H, Rose HJ, Grace AA (2001). Chronic cold stress reduces the spontaneous activity of ventral tegmental dopamine neurons. Neuropsychopharmacology 24: 410-419. 
Nestler EJ, Carlezon WA Jr (2006). The mesolimbic dopamine reward circuit in depression. Biol Psychiatry 59: 1151-1159.

Patton MH, Bizup BT, Grace AA (2013). The infralimbic cortex bidirectionally modulates mesolimbic dopamine neuron activity via distinct neural pathways. J Neurosci 33: 16865-16873.

Ressler KJ, Nemeroff CB (2000). Role of serotonergic and noradrenergic systems in the pathophysiology of depression and anxiety disorders. Depress anxiety 12(Suppl 1): 2-19.

Salamone JD, Correa M (2012). The mysterious motivational functions of mesolimbic dopamine. Neuron 76: 470-485.

Sartorius A, Kiening KL, Kirsch P, von Gall CC, Haberkorn U, Unterberg AW et al (2010). Remission of major depression under deep brain stimulation of the lateral habenula in a therapyrefractory patient. Biol Psychiatry 67: e9-e11.

Slattery DA, Cryan JF (2012). Using the rat forced swim test to assess antidepressant-like activity in rodents. Nat Protocols 7: 1009-1014.

Stamatakis AM, Stuber GD (2012). Activation of lateral habenula inputs to the ventral midbrain promotes behavioral avoidance. Nat Neurosci 15: 1105-1107.

Stopper CM, Floresco SB (2014). What's better for me? Fundamental role for lateral habenula in promoting subjective decision biases. Nat Neurosci 17: 33-35.

Treadway MT, Zald DH (2011). Reconsidering anhedonia in depression: lessons from translational neuroscience. Neurosci Biobehav Rev 35: 537-555.
Tye KM, Mirzabekov JJ, Warden MR, Ferenczi EA, Tsai HC, Finkelstein J et al (2013). Dopamine neurons modulate neural encoding and expression of depression-related behaviour. Nature 493: $537-541$.

Ungless MA, Grace AA (2012). Are you or aren't you? Challenges associated with physiologically identifying dopamine neurons. Trends Neurosci 35: 422-430.

Valenti O, Gill KM, Grace AA (2012). Different stressors produce excitation or inhibition of mesolimbic dopamine neuron activity: response alteration by stress pre-exposure. Eur J Neurosci 35: 1312-1321.

Valenti O, Lodge DJ, Grace AA (2011). Aversive stimuli alter ventral tegmental area dopamine neuron activity via a common action in the ventral hippocampus. J Neurosci 31: 4280-4289.

Weiss JM (1997). Does decreased sucrose intake indicate loss of preference in CMS model? Psychopharmacology 134: 368-370

Willner P, Lappas S, Cheeta S, Muscat R (1994). Reversal of stress-induced anhedonia by the dopamine receptor agonist, pramipexole. Psychopharmacology 115: 454-462.

Willner P, Muscat R, Papp M (1992). Chronic mild stress-induced anhedonia: a realistic animal model of depression. Neurosci Biobehav Rev 16: 525-534.

Zimmerman EC, Grace AA (2016). The nucleus reuniens of the midline thalamus gates prefrontal-hippocampal modulation of ventral tegmental area dopamine neuron activity. J Neurosci 36: $8977-8984$.

Supplementary Information accompanies the paper on the Neuropsychopharmacology website (http://www.nature.com/npp) 